\title{
Erratum to: A Trace-Time Framework for Prediction of Elevation Angle Over Land Mobile LEO Satellites Networks
}

\section{Younes Seyedi · Fazel Rahimi}

Published online: 26 May 2011

(C) Springer Science+Business Media, LLC. 2011

\section{Erratum to: Wireless Pers Commun DOI 10.1007/s11277-010-0094-5}

Equations (12) and (17) are incorrect in the original publication.

(I) Equation (12):

$$
\theta(t)=\tan ^{-1}\left(\frac{\cos \left(\left(\omega_{E} \cos i-\omega_{S}\right) t+\psi_{0}\right) \times \cos \gamma_{m}-\frac{R e}{R}}{\sin \left(\cos \left(\left(\omega_{E} \cos i-\omega_{S}\right) t+\psi_{0}\right) \times \cos \gamma_{m}\right)}\right)
$$

Should change to:

$$
\theta(t)=\tan ^{-1}\left(\frac{\cos \left(\left(\omega_{E} \cos i-\omega_{S}\right) t+\psi_{0}\right) \times \cos \gamma_{m}-\frac{R e}{R}}{\sin \left(\cos ^{-1}\left(\cos \left(\left(\omega_{E} \cos i-\omega_{S}\right) t+\psi_{0}\right) \times \cos \gamma_{m}\right)\right)}\right)
$$

The online version of the original article can be found under doi:10.1007/s11277-010-0094-5.

Y. Seyedi $(\varangle)$

Department of Electrical and Computer Engineering, University of Tehran, Tehran, Iran e-mail: yseyedi@ieee.org

F. Rahimi

Department of Aerospace Engineering, Sharif University of Technology, Tehran, Iran e-mail: rahimi.fazel@gmail.com 
(II) Equation (17):

$$
\theta(t)=\tan ^{-1}\left(\frac{\cos \left\{\left(\omega_{E} \cos i-\omega_{S}\right) t+\cos ^{-1}\left(\frac{\cos \left(\cos ^{-1}\left(\frac{R e}{R} \cos \theta_{C}\right)-\theta_{C}\right)}{\cos \gamma_{m}}\right)\right\} \times \cos \gamma_{m}-\frac{R e}{R}}{\sin \left\{\cos \left\{\left(\omega_{E} \cos i-\omega_{S}\right) t+\cos ^{-1}\left(\frac{\cos \left(\cos ^{-1}\left(\frac{R e}{R} \cos \theta_{C}\right)-\theta_{C}\right)}{\cos \gamma_{m}}\right)\right\} \times \cos \gamma_{m}\right\}}\right)
$$

Should change to:

$$
\theta(t)=\tan ^{-1}\left(\frac{\cos \left\{\left(\omega_{E} \cos i-\omega_{S}\right) t+\cos ^{-1}\left(\frac{\cos \left(\cos ^{-1}\left(\frac{R e}{R} \cos \theta_{C}\right)-\theta_{C}\right)}{\cos \gamma_{m}}\right)\right\} \times \cos \gamma_{m}-\frac{R e}{R}}{\sin \left\{\cos ^{-1}\left\{\cos \left\{\left(\omega_{E} \cos i-\omega_{S}\right) t+\cos ^{-1}\left(\frac{\cos \left(\cos ^{-1}\left(\frac{R e}{R} \cos \theta_{C}\right)-\theta_{C}\right)}{\cos \gamma_{m}}\right)\right\} \times \cos \gamma_{m}\right\}\right]}\right)
$$

